\title{
Manual de prácticas de laboratorio de tecnologías aplicadas a la logística
}

\author{
Myrna Hortencia Lezama León \\ mlezama@upmh.edu.mx \\ Eduardo Cruz Aldana \\ ealdana@upmh.edu.mx \\ Hector Armando Figueroa Urrea \\ hfigueroa@upmh.edu.mx \\ Rafael Ordoñez Gutiérrez \\ rordonez@upmh.edu.mx
}

Universidad Politécnica Metropolitana de Hidalgo

Tolcayuca - México

\section{RESUMEN}

Este manual de prácticas ha sido desarrollado para el Laboratorio de Tecnologías Aplicadas a la Logística (LTAL), el cual está a cargo de la Ingeniería en Logística y Transporte de la Universidad Politécnica Metropolitana de Hidalgo (UPMH). Este laboratorio tiene como objetivo el proveer a los alumnos de la carrera en Ingeniería en Logística y Transporte de la UPMH, de espacios para poner en práctica los conocimientos adquiridos en el aula, aplicando tecnologías de vanguardia de la Industria 4.0, además de cumplir con las Líneas de Generación Aplicada del Conocimiento (LGAC) de la carrera, se abarcan las metodologías Justo a Tiempo, Planeación del requerimiento de materiales y análisis de tiempos.

Palabras clave: prácticas de laboratorio; logística; industria 4.0. 


\title{
Laboratory practice manual of technologies applied to logistics
}

\begin{abstract}
This manual of practices has been developed for the Laboratory of Technologies Applied to Logistics (LTAL), which is in charge of the Engineering in Logistics and Transportation of the Universidad Politécnica Metropolitana de Hidalgo (UPMH). The objective of this laboratory is to provide students of the degree in Engineering in Logistics and Transportation at UPMH, with spaces to put into practice the knowledge acquired in the classroom, applying cutting-edge technologies of Industry 4.0, in addition to complying with the Lines of Applied Generation of Knowledge (LGAC) of the career, the methodologies Just in Time, planning of the requirement of materials and time analysis are covered.
\end{abstract}

Keywords: laboratory practices; logistics; industry 4.0 .

Artículo recibido: 02 noviembre. 2021 Aceptado para publicación: 28 noviembre 2021

Correspondencia: mlezama@upmh.edu.mx Conflictos de Interés: Ninguna que declarar 


\section{INTRODUCCIÓN}

Este manual de prácticas de laboratorio se ha elaborado para la carrera de Ingeniería en Logística y Transporte (ILT), de la Universidad Politécnica Metropolitana de Hidalgo (UPMH), ubicada en Tolcayuca, Hidalgo, pertenece al sistema de Universidades Politécnicas en México. Esta universidad tiene como objetivo “impartir educación superior en los niveles de licenciatura, ingeniería, especialización tecnológica y otros estudios de posgrado, así como cursos de actualización en sus diversas modalidades, para preparar profesionales con una sólida formación científica, tecnológica y en valores cívicos y éticos". (UPMH, 2021)

La logística se ha convertido en uno de los principales centros de atención para las empresas tanto locales, nacionales, así como internacionales debido a la importancia que representan para ser altamente competitivas en el mercado. De acuerdo con Pinheiro et al. (2017), se entiende por logística a “... la gestión estratégica de la adquisición, traslado y almacenaje de materiales y productos acabados, sus informaciones relacionadas, mediante canales de distribución, maximizando el lucro presente y futuro".

El programa educativo en ILT, tiene como objetivo: "Formar profesionales que sean capaces de diagnosticar, planear, diseñar y optimizar soluciones integrales de ingeniería a las problemáticas de los sistemas logísticos y de transporte de las organizaciones". (UPMH, 2021)

Es por ello que con este manual de prácticas se busca generar profesionales de la logística más preparados.

\subsection{Problemática}

Uno de los objetivos de las universidades es crear competencias profesionales a través de su formación académica, por ello, es que cuentan con un perfil de egreso que muestra las habilidades y competencias que debieron ser adquiridas a lo largo de la carrera. En la UPMH estas competencias son adquiridas a través del aprendizaje en el aula y puestas en práctica en espacios como "Estancias y Estadías".

Las estancias y estadías son procesos formativos no escolarizados que se llevan a cabo durante el $4^{\circ}, 7^{\circ}$ y $10^{\circ}$ cuatrimestre de la carrera, y tienen por objetivo poner en práctica los conocimientos y competencias adquiridas durante sus ciclos de formación, dichas estancias y estadía se realizan en un ambiente real de trabajo, ya sea en organizaciones del sector público o privado. 
Aunque se cuentan con estos espacios, muchas veces no son suficientes para reforzar las competencias adquiridas, por ello, es importante que dentro de las aulas se genere la práctica por medio de diversos talleres o laboratorios.

En específico para la carrera de ILT, se requiere de la práctica de diversas asignaturas para que los alumnos observen el funcionamiento de espacios de trabajo, los cuales muchas veces, por no contar los estudiantes con experiencia previa en almacenes o procesos productivos, les es difícil asimilar la información que reciben de forma teórica, así como en otros casos, pierden de vista el impacto de las decisiones tomadas en los diferentes procesos.

\subsection{Objetivo General}

- Ser una guía para que los alumnos de la UPMH de la carrera en ILT adquieran y refuercen las competencias necesarias en su formación académica a través de las prácticas en el Laboratorio de Tecnologías Aplicadas a la Logística.

\subsubsection{Objetivos Específicos}

1) El alumno realizará estudios de tiempos y movimientos de operaciones.

2) El alumno realizará balanceo de líneas de ensamble.

3) El alumno podrá implementar un sistema justo a tiempo.

4) El alumno implementará sistemas de planeación de requerimiento de materiales para líneas de ensamble.

5) El alumno podrá aplicar sistemas de rastreabilidad utilizando códigos de barras.

\subsection{Justificación}

Las prácticas de laboratorio son actividades didácticas que sirven para reforzar conceptos, métodos y tecnologías que se han visto de forma teórica (Suárez y Mendoza, 2006), por lo que los laboratorios son herramientas esenciales en el proceso de aprendizaje de las ingenierías como también en la investigación y la industria. En el sector educativo la experiencia en laboratorios también brinda la valiosa oportunidad para que los estudiantes desarrollen, habilidades de comunicación, tanto oral como escrita, así como el liderazgo y cooperación.

Además, la dinámica educativa va cambiando y adaptándose a las necesidades de los sectores laborales, por lo que el concepto de enseñanza-aprendizaje de ser un método de observación pasa a convertirse en un modelo orientado a la participación de estudiantes y docentes en casos explícitos en su área y campo de desarrollo. La falta de laboratorios 
que manejen herramientas tecnológicas de alto impacto como software, hardware, simuladores, entre otros, puede generar debilidades en el desarrollo integral de los estudiantes como la falta de habilidades prácticas, capacidad para resolver problemas y liderazgo.

De acuerdo con Insausti (2020), las prácticas de laboratorio son una gran alternativa para plasmar los cimientos teóricos en actividades ejecutables y el desarrollo de competencias de los estudiantes; y de esta manera, aprovechar su participación y así puedan desarrollar al máximo todas sus capacidades, aumentando su curiosidad, investigación, observación, creatividad, etc., lo que convierte a los estudiantes en jóvenes con visión y emprendimiento, cuyo aprendizaje no queda solo en las aulas de clase, y les permita adquirir diferentes habilidades como:

- Resolución de conflictos

- Planificación y organización

- Habilidades comunicativas

- Análisis de datos

- Uso de programas informáticos

- Creatividad

- Persuasión

Por otra parte, es importante profundizar en estos temas puesto que son acciones por realizar en cualquier cargo a desempeñar como futuros profesionales, por ejemplo, la simulación en gestión de inventarios o líneas de ensamble, puede ser clave en la reducción de costos y tiempos y por lo tanto presentar un impacto significativo en el desempeño laboral de un ingeniero en logística.

El presente manual tiene como finalidad orientar y aportar herramientas de aprendizaje y apoyo para los estudiantes de la carrera de Ingeniería en Logística y Transporte, a través de prácticas que instruyan, capacitan y generan competencias que les permita llevar los conocimientos teóricos a la práctica y comprensión de los procesos logísticos de las empresas y su conexión con la industria 4.0.

El manual de prácticas de laboratorio es un apoyo para cumplir con los objetivos y lograr las competencias requeridas a lo largo de la carrera, ya que, será un gran impulso a la formación profesional y desarrollo dentro del campo laboral. La

Tabla 1 
Tabla 1 muestra la relación del cumplimiento de las competencias que deberán adquirir los estudiantes de ILT a través de los objetivos planteados en este manual.

\section{Tabla 1}

Cumplimiento de competencias con los objetivos

\section{Objetivos Específicos Competencias}

a) El alumno realizará estudios de Tiempos y movimientos de operaciones

Gestionar la producción de acuerdo con la capacidad de la planta para cumplir con la demanda.

b) El alumno realizará Balanceo de Líneas de ensamble

Gestionar la producción de acuerdo con la capacidad de la planta para cumplir con la demanda.

c) El alumno podrá implementar un sistema

Determinar los planes de adquisición de acuerdo con las necesidades de producción para determinar el programa de abastecimiento

JIT

Gestionar la producción de acuerdo con la capacidad de la planta para cumplir con la demanda.

Controlar los inventarios mediante la clasificación y empleo de TIC's con el fin de cumplir con los requerimientos de los clientes internos y externos.

Gestionar la producción de acuerdo con la

d) El alumno implementará sistemas MRP capacidad de la planta para cumplir con la en líneas de ensamble demanda.

Controlar los inventarios mediante la clasificación y empleo de TIC's con el fin de cumplir con los requerimientos de los clientes internos y externos.

e) El alumno podrá aplicar sistemas de Controlar los inventarios mediante la clasificación rastreabilidad utilizando código de barras y empleo de TIC's con el fin de cumplir con los requerimientos de los clientes internos y externos.

Nota. Elaboración propia, basado en UPMH (2021).

\subsection{Marco teórico}

\subsubsection{Importancia de las prácticas en el ámbito profesional}

Hoy en día, de acuerdo con las investigaciones efectuadas por Rodríguez, Armengol y Meneses (2017), es importante el realizar estudios que analicen la relación de características conforme al impacto que producen dentro de una atmósfera de trabajo, en este caso, las prácticas en la vida profesional, ya que la aplicación de periodos de prácticas curriculares durante un cierre de formación académica, se consideran periodos fundamentales para un correcto desarrollo competencial y profesional, ya que ayuda a comprender con mayor profundidad elementos que son claves para un éxito profesional. De igual manera, destaca el papel que desempeña el tutor en el desarrollo de prácticas, puesto que se refleja un mayor desenvolvimiento de los practicantes con base en toda la 
guía proporcionada en el desarrollo, centralizado en “...el apoyo psicológico y emocional recibido durante sus prácticas" (Sorensen, 2014).

\subsubsection{Laboratorios en la educación}

Como la mayoría de las actividades que realiza el ser humano, las prácticas en el ámbito académico-profesional se han identificado con diferentes nombres, tales como: trabajo de laboratorio, trabajo práctico, prácticas experimentales, entre otros; algunos de estos términos aún son empleados en algunas locaciones, sin embargo, el término que es mayormente ubicado es el de "prácticas". "Las prácticas no deben limitarse a un espacio físico" Marín (2008), esta idea nos permite tener un panorama más amplio de que la aplicación no es lo único que importa dentro de una práctica, sino también el entendimiento de lo que se está realizando, así como la aplicación dentro de un panorama personal-profesional. Para alcanzar una explotación al máximo de una práctica, de acuerdo con Marín, se sugiere la implementación de actividades dinámicas que pongan en práctica las destrezas de los estudiantes, a fin de evitar un ambiente tedioso de trabajo que no dé muestras reales de aprendizaje.

Espinosa, González y Hernández (2016), presentan el impacto de las prácticas dentro de un proceso de educación formal, haciendo hincapié en la importancia del papel que juegan los docentes al momento de realizar su aplicación, ya que éste debe manejar una organización que tome en ponderación tanto los tiempos como los espacios en los que se debe desarrollar la práctica, presentando etapas que permitan un mayor entendimiento de las mismas, y, que, a su vez, permitan a los alumnos a interactuar con la actividad en sí, a fin de que vayan descubriendo herramientas propias para la aplicación futura de la práctica. Hoy en día, es bien sabido que cada grupo de estudiantes se conformará de distinta manera partiendo de las personalidades de cada miembro, por lo que esto último será el factor clave para el desarrollo de la planificación, para que se permita ver a los estudiantes no solo el reflejo de la actividad en curso, si no la manera en la que se ha construido o en su defecto, se está construyendo un conocimiento científico.

\section{ESTRATEGIAS METODOLÓGICAS O MATERIALES Y MÉTODOS}

La elaboración de este manual de prácticas tiene un enfoque cualitativo debido a que existe "la recolección de datos sin medición numérica" (Fernández, Baptista y Hernández, 2014). Además, se basó en una metodología para el desarrollo de competencias (Exactas, 
2010), se adaptan los elementos que deben tener las prácticas, establecidos por Suárez y Mendoza (2016), como son:
a) Título
b) Introducción
c) Objetivo
d) Metodología
e) Recursos, materiales y equipo
f) Descripción del desarrollo de la práctica
g) Evaluación
h) Bibliografía
i) Resultados y conclusiones

Cabe resaltar que el fundamento metodológico se observa de forma amplia en el programa académico de las asignaturas por lo que no se presenta en este manual, además de que los tres últimos apartados como son evaluación, bibliografía y resultados se manejan al final de forma general para todas las prácticas.

Finalmente, la mayor parte de las prácticas aquí presentadas se han generado desde la experiencia de los autores y algunas se obtuvieron de material didáctico disponible.

\section{RESULTADOS Y DISCUSIÓN}

En este apartado se presentan las diversas prácticas que abarca el manual.

\section{PRÁCTICA 1. Cálculo de suplementos}

\section{Introducción}

Los suplementos se refieren al tiempo de compensación para el trabajo, debido a retrasos, demoras o elementos contingentes que son parte de la tarea de un trabajador (Criollo, 2005).

\section{Objetivo de la práctica}

- Identificar los suplementos a considerar dentro de la tarea a estudiar.

\section{Recursos Materiales}

- Libreta de anotación

- Tabla de suplementos (Tabla 2)

- Formato de suplementos (Tabla 2 
Suplementos y porcentajes de tiempo normales

\begin{tabular}{|c|c|c|c|c|c|c|c|}
\hline \multicolumn{8}{|c|}{ SISTEMA DE SUPLEMENTOS POR DESCANSO EN PORCENTAIE DE LOS TIEMPOS NOMALES } \\
\hline \multicolumn{8}{|c|}{ 1. SUPLEMENTO CONSTANTES } \\
\hline $\mathrm{N}^{*}$ & Tipo de Suplemento & Hombres & Mujeres & & & & \\
\hline A. & $\begin{array}{l}\text { Suplemento por necesidades } \\
\text { personales }\end{array}$ & 5 & 7 & & & & \\
\hline B. & Suplemento base por fatiga & 4 & 4 & & & & \\
\hline \multicolumn{8}{|c|}{ 2. SUPLEMENTO VARIABLES } \\
\hline $\mathrm{N}^{*}$ & Tipo de Suplemento & Hombres & Mujeres & $\mathrm{N}^{*}$ & Tipo de Suplemento & Hombres & Mujeres \\
\hline A. & Suplemento por trabajar de pie & 2 & 4 & F. & Concentración intensa & & \\
\hline \multirow[t]{4}{*}{ B. } & Suplemento por postura anormal & & & & Trabajos de cierta precisión & 0 & 0 \\
\hline & Ligeramente incómoda & 0 & 1 & & Trabajos precisos o fatigosos & 2 & 2 \\
\hline & Incómoda (indinado) & 2 & 3 & & $\begin{array}{l}\text { Trabajo de gran precisión o muy } \\
\text { fatigosos }\end{array}$ & 5 & 5 \\
\hline & Muy incómoda (echado, estirado) & 7 & 7 & G. & Ruido & & \\
\hline \multirow[t]{8}{*}{ c. } & $\begin{array}{l}\text { Uso de Fuerza/energía muscular } \\
\text { (Levantar, tirar, empujar) }\end{array}$ & & & & Continuo & 0 & $\underline{0}$ \\
\hline & Peso levantado $[\mathrm{Kg}]$ & & & & Intermitente $y$ fuerte & 2 & 2 \\
\hline & 2,5 & 0 & 1 & & Intermitente y muy fuerte & 5 & 5 \\
\hline & 5 & 1 & 2 & & Estridente y fuerte & & \\
\hline & 10 & 3 & 4 & H. & Tensión mental & & \\
\hline & 25 & 9 & 20 & & Proceso bastante complejo & 1 & 1 \\
\hline & & & $\operatorname{Max}$ & & $\begin{array}{l}\text { Proceso complejo o atención } \\
\text { dividida entre muchos objetos }\end{array}$ & 4 & 4 \\
\hline & 35,5 & 22 & & & Muy complejo & 8 & 8 \\
\hline \multirow[t]{4}{*}{ D. } & Mala lluminación & & & I. & Monotonía & & \\
\hline & $\begin{array}{l}\text { Ligeramente por debajo de la } \\
\text { potencia calculada }\end{array}$ & 0 & 0 & & Trabajo algo monótono & 0 & 0 \\
\hline & Bastante por debajo & 2 & 2 & & Trabajo bastante monótono & 1 & 1 \\
\hline & Absolutamente insuficiente & 5 & 5 & & Trabajo muy monótono & 4 & 4 \\
\hline \multirow[t]{6}{*}{ E. } & Condiciones atmosféricas & & & J. & Tedio & & \\
\hline & Indice de enfriamiento Kata & & & & Trabajo algo aburrido & 0 & 0 \\
\hline & 16 & \multicolumn{2}{|c|}{0} & & Trabajo bastante aburrido & 2 & 1 \\
\hline & 8 & \multicolumn{2}{|c|}{10} & & Trabajo muy aburrido & 5 & 2 \\
\hline & 4 & \multirow{2}{*}{\multicolumn{2}{|c|}{$\frac{45}{100}$}} & & & & \\
\hline & 2 & & & & & & \\
\hline
\end{tabular}

- Tabla 3)

Fuente: Criollo (2005).

\section{Desarrollo}

- Determina los suplementos que son necesarios a considerar en la siguiente situación.

María trabaja en un Call Center en la Ciudad de México con un horario de 8:00 a 16:00

hrs de lunes a sábado. Su actividad primordial es recibir llamadas de clientes en todo momento por lo que se ve obligada a permanecer sentada la mayor parte de su jornada además de que no se le concede más de 10 minutos cada 3 horas para sus necesidades básicas como lo son: idas al sanitario, comer un refrigerio o descanso de postura. Debido a que todas las llamadas deben ser registradas en el sistema María mantiene una postura inclinada y con constante atención al monitor. Por el reducido espacio de la oficina donde labora, se encuentra trabajando con 4 compañeros en el mismo cubículo donde todos contestan llamadas continuamente por lo tanto muchas veces es difícil concentrarse por todo el ruido a su alrededor. 
Registra en la tabla 3 los suplementos que consideres acordes a la situación, así como su valor y la sumatoria final.

Tabla 2

Suplementos y porcentajes de tiempo normales

\begin{tabular}{|c|c|c|c|c|c|c|c|}
\hline \multicolumn{8}{|c|}{ SISTEMA DE SUPLEMENTOS POR DESCANSO EN PORCENTAIE DE LOS TIEMPOS NOMALES } \\
\hline \multicolumn{8}{|c|}{ 1. SUPLEMENTO CONSTANTES } \\
\hline $\mathrm{N}^{*}$ & Tipo de Suplemento & Hombres & Mujeres & & & & \\
\hline A. & $\begin{array}{l}\text { Suplemento por necesidades } \\
\text { personales }\end{array}$ & 5 & 7 & & & & \\
\hline B. & Suplemento base por fatiga & 4 & 4 & & & & \\
\hline \multicolumn{8}{|c|}{ 2. SUPLEMENTO VARIABLES } \\
\hline $\mathrm{N}^{*}$ & Tipo de Suplemento & Hombres & Mujeres & $\mathrm{N}^{\circ}$ & Tipo de Suplemento & Hombres & Mujeres \\
\hline A. & Suplemento por trabajar de pie & 2 & 4 & F. & Concentración intensa & & \\
\hline \multirow[t]{4}{*}{ B. } & Suplemento por postura anormal & & & & Trabajos de dierta precisión & 0 & 0 \\
\hline & Ligeramente incómoda & o & 1 & & Trabajos precisos o fatigosos & 2 & 2 \\
\hline & Incómoda (indinado) & 2 & 3 & & $\begin{array}{l}\text { Trabajo de gran precisión o muy } \\
\text { fatigosos }\end{array}$ & 5 & 5 \\
\hline & Muy incómoda (echado, estirado) & 7 & 7 & G. & Ruido & & \\
\hline \multirow[t]{8}{*}{ c. } & $\begin{array}{l}\text { Uso de Fuerza/energía muscular } \\
\text { (Levantar, tirar, empujar) }\end{array}$ & & & & Continuo & 0 & $\underline{0}$ \\
\hline & Peso levantado $[\mathrm{Kg}]$ & & & & Intermitente y fuerte & 2 & 2 \\
\hline & 2,5 & 0 & 1 & & Intermitente y muy fuerte & 5 & 5 \\
\hline & 5 & 1 & 2 & & Estridente y fuerte & & \\
\hline & 10 & 3 & 4 & H. & Tensión mental & & \\
\hline & 25 & 9 & 20 & & Proceso bastante complejo & 1 & 1 \\
\hline & & & $\operatorname{Max}$ & & $\begin{array}{l}\text { Proceso complejo o atención } \\
\text { dividida entre muchos objetos }\end{array}$ & 4 & 4 \\
\hline & 35,5 & 22 & & & Muy complejo & 8 & 8 \\
\hline \multirow[t]{4}{*}{ D. } & Mala lluminación & & & I. & Monotonía & & \\
\hline & $\begin{array}{l}\text { Ligeramente por debajo de la } \\
\text { potencia calculada }\end{array}$ & 0 & 0 & & Trabajo algo monótono & 0 & 0 \\
\hline & Bastante por debajo & 2 & 2 & & Trabajo bastante monótono & 1 & 1 \\
\hline & Absolutamente insuficiente & 5 & 5 & & Trabajo muy monótono & 4 & 4 \\
\hline \multirow[t]{6}{*}{ E. } & Condiciones atmosféricas & & & J. & Tedio & & \\
\hline & Indice de enfriamiento Kata & & & & Trabajo algo aburrido & 0 & 0 \\
\hline & 16 & \multicolumn{2}{|c|}{0} & & Trabajo bastante aburrido & 2 & 1 \\
\hline & 8 & \multirow{2}{*}{\multicolumn{2}{|c|}{10}} & & Trabajo muy aburrido & 5 & 2 \\
\hline & 4 & & & & & & \\
\hline & 2 & \multicolumn{2}{|c|}{100} & & & & \\
\hline
\end{tabular}

Tabla 3

Fuente: Criollo (2005).

Formato de suplementos

\begin{tabular}{|l|l|}
\hline \multicolumn{1}{|c|}{ Suplemento } & Valor \\
\hline & \\
\hline Sumatoria & \\
\hline
\end{tabular}

\section{PRÁCTICA 2. Estudio de tiempos}

\section{Introducción}

Según Kanawaty (1996), el estudio de tiempos y movimientos consiste en el registro y el examen crítico de los modos de realizar actividades con el fin de mejorar y simplificar las 
tareas, establecer mejores formas de realización y así determinar el tiempo que invierte un trabajador calificado en llevar a cabo una tarea según una norma de ejecución preestablecida.

\section{Objetivo de la práctica}

- Aplicar las técnicas de medición de tiempos de operaciones, analizando los factores y los elementos que intervienen en el tiempo trabajo.

\section{Recursos materiales}

- Set de lego con instructivo

- 1 cronómetro

- Formato de estudio de tiempos (Tabla 4)

- Dispositivo de grabación o cámara de video

\section{Desarrollo}

En equipos de cuatro personas mínimo, cada uno tomará uno de los siguientes roles: operador, cronometrista, camarógrafo y supervisor encargado del llenado del formato. Preparar tu mesa de trabajo de manera que tengas todas las piezas del set visibles, suficiente espacio para el ensamblado, así como los materiales antes mencionados. Es importante tener a la mano el instructivo del set para el ensamblado. El cronometrista iniciara a contar el tiempo mientras que el encargado de llenar el formato hace cualquier anotación que le parezca importante para la valoración del operador, tiempos y suplementos para registrar todos los datos necesarios para el cálculo del tiempo estándar. Es recomendable repetir el ensamble y el conteo del tiempo por lo menos 5 veces para tener una buena base de datos a evaluar.

\section{Tabla 4}

Formato para estudio de tiempos 


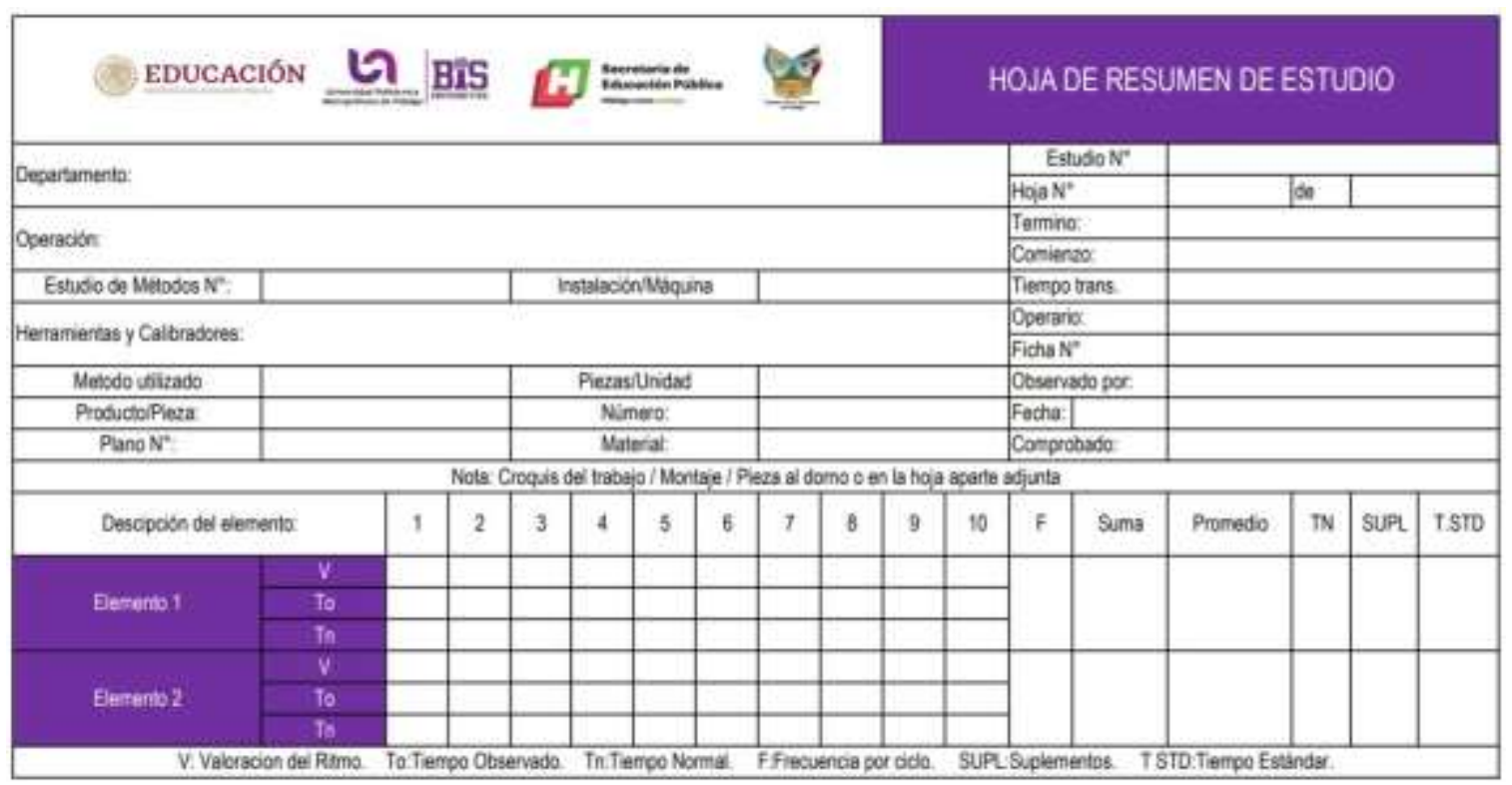

\section{PRÁCTICA 3: Diagrama de precedencias}

\section{Introducción}

El diagrama de precedencias es la representación de cada tarea individual de un proceso y se aplica al realizar balanceo de líneas de producción (Niebel, 2009).

\section{Objetivo de la práctica}

- El alumno realizará un diagrama de precedencias de un proceso.

\section{Recursos materiales}

- Set de lego con instructivo

- Estudio de tiempos de la práctica 2.

\section{Desarrollo}

- De acuerdo con el instructivo del set de lego, elaborar un diagrama de precedencias para el ensamble final del producto.

\section{PRÁCTICA 4: Balanceo de líneas de producción}

\section{Introducción}

El balanceo de líneas consiste en la correcta asignación de recursos a los procesos de fabricación (maquinaria y personal), cumpliendo con la producción requerida. Su objetivo principal es igualar los tiempos de trabajo (correspondientes a cada una de las estaciones de producción que la línea contenga) en el proceso de producción y así lograr un equilibrio que ayude a minimizar costos, mantener la calidad y usar el tiempo eficientemente (Chase, Jacobs \& Aquilano, 2009).

\section{Objetivo de la práctica}


- El alumno realizará un balanceo de líneas.

\section{Recursos materiales}

- Set de lego con instructivo

- Estudio de tiempos de la práctica 2.

- Diagrama de precedencias práctica 3.

\section{Desarrollo}

Con la información obtenida en las prácticas señaladas y los siguientes datos, elaborar el balanceo de líneas.

En una planta de ensamble de carritos, existen 400 minutos disponibles para producir la unidad manual en esta planta y la demanda promedio es de 80 unidades por día. Establece:

a) Tiempo ciclo

b) Mínimo número de estaciones de trabajo

c) Porcentaje de eficiencia

d) Número de trabajadores

e) Asignación de operaciones por estaciones de trabajo.

\section{PRÁCTICA 5: Simulación del sistema de producción}

\section{Introducción}

En la industria 4.0 se hace uso de tecnologías para agilizar los procesos productivos, tal es el caso de equipo de avance automático como las bandas transportadoras.

\section{Objetivo de la práctica}

- Los alumnos comprobarán lo establecido en el balanceo de líneas aplicando sistemas de avance continuo de materiales.

\section{Recursos materiales}

- Set de Lego con instructivo

- Balanceo de líneas de la práctica 4

- Banda transportadora de flujo continuo.

\section{Desarrollo}

La cantidad de participantes será de uno por cada estación de trabajo de acuerdo con la información obtenida en la práctica señalada, quienes fungirán como los operarios de cada estación. 
Acomodar las bandas transportadoras en línea y regular la velocidad de acuerdo con el tiempo ciclo y la distancia entre las estaciones de trabajo.

Simular la línea de ensamble y hacer los ajustes necesarios para su correcto funcionamiento.

Hacer una reflexión sobre los resultados obtenidos.

\section{PRÁCTICA 6: Producción con sistema tipo arrastre}

\section{Introducción}

La metodología Justo a Tiempo (JIT, por sus siglas en inglés), debe su nombre a la filosofía de realizar sólo lo que es necesario, cuando es necesario y en la cantidad que es necesaria. Ni antes, lo que provocaría acumulación de inventario o de productos inacabados, ni después, lo que implicaría un retraso en el servicio a los clientes y su correspondiente aumento de insatisfacción (Blandón, 2021).

\section{Objetivo de la práctica}

- El alumno verificará el uso de las tarjetas Kanban y su importancia dentro de un sistema JIT.

\section{Recursos materiales}

- Bloques de Lego

- Formato tarjeta Kanban (Tabla 5)

\section{Desarrollo}

Elegir una figura que será el producto final, esta debe contener un mínimo de 6 figuras para llevarlas a cabo dentro del área de producción.

Se deben considerar como mínimo 5 áreas de trabajo; 3 de ensamble, 1 de producto terminado/cliente y 1 de proveedor.

Se requiere de 6 integrantes, los cuales asumirán los siguientes roles:

- Cliente

- Encargado PT

- Encargado de área 3

- Encargado de área 2

- Encargado de área 1

- Proveedor/MP 
En la primer área debe estar el encargado del producto terminado, mismo que le entregará al cliente la cantidad que éste requiera; en este caso consideremos que serán dos piezas las que solicitó el cliente y se despachan.

Ahora, vemos que ha quedado vacío el lote de existencias mínimas en el área de producto terminado, por lo que debemos solicitar al área anterior un lote de PT por medio de una tarjeta Kanban (Tabla 5), de manera que le dejamos una tarjeta en el área anterior (en este caso: área 3 de ensamble) solicitando el lote.

\section{Tabla 5}

Tarjeta Kanban de producción

\begin{tabular}{|c|l|}
\hline Proceso & \\
\hline Depositar piezas en: & \\
\hline Referencia: & \\
\hline Nombre de la pieza: & \\
\hline Cantidad a producir: & \\
\hline
\end{tabular}

Fuente: Salazar (2019).

El área 3, ensamblará su lote y pasará el PT al área de PT para el momento en que el cliente lo requiera y al mismo tiempo generará una tarjeta para el área 2 solicitando suministro de su material o producto en proceso.

El área 2 pasará el WIP, que en esta área se trabaja, al área 3 y de la misma manera el área 2 generará una tarjeta Kanban para solicitar suministro del área 1 de ensamble.

El área 1 pasará su lote trabajado al área 2 (solicitante) y después debe comenzar a trabajar su nuevo lote, para ello generará una tarjeta Kanban al área anterior (en este caso el proveedor o almacén de materia prima) para que se le pueda suministrar material y trabajar el producto.

\section{PRÁCTICA 7. Elaboración de un diagrama de árbol}

\section{Introducción}

La lista de materiales (BOM, por sus siglas en inglés) es necesaria para conocer de cada artículo su estructura de fabricación, en donde quedan reflejados los diferentes elementos que lo componen, así como el número necesario de cada uno de esos elementos para fabricar una unidad de este artículo. Esta información suele ser representada en forma de árbol donde el nodo raíz (principal) representa el artículo que se describe, saliendo de él un nodo para cada uno de sus componentes; en el nodo se indica el nombre del 
componente y el número de unidades necesarias para elaborar una unidad de artículo descrito (Rodríguez y León, s.f.).

\section{Objetivo de la práctica}

- El alumno podrá estructurar el diagrama de árbol de un producto a partir de su lista de materiales.

\section{Recursos materiales}

- Hoja de cálculo (Excel)

- Set de Lego con instructivo.

\section{Desarrollo}

Identificar la lista de materiales del set de Lego y de acuerdo con la secuencia de operaciones (se puede hacer uso del balanceo de líneas de la práctica 4), armar el diagrama de árbol de los materiales.

\section{PRÁCTICA 8. MRP de Lego}

\section{Introducción}

Los sistemas de Planeación de Requerimiento de Materiales (MRP por sus siglas en inglés), se refieren a la identificación de los materiales a utilizar en cada etapa del proceso, estableciendo la cantidad y el momento en específico que se requiere en un proceso en específico, todo esto de acuerdo con el plan de producción (Chase et al., 2009).

\section{Objetivo de la práctica}

- Realizar la planificación de requerimientos de materiales para un plan de producción de carritos Lego.

\section{Recursos materiales}

- Hoja de cálculo (Excel)

- Formato de tablas MRP

- Diagrama de árbol práctica 7

- Balanceo de líneas práctica 4

\section{Desarrollo}

Elaborar el MRP para la línea de ensamble de Lego (práctica 4), estableciendo los contenedores a requerir por cada área de trabajo de acuerdo con la cantidad de piezas a producir según las necesidades de la línea.

\section{PRÁCTICA 9. Simulación del MRP en una línea de ensamble}




\section{Introducción}

La mejor forma de observar la eficacia de un análisis es a través de su puesta en marcha y la simulación del proceso de producción con avance continuo, es decir, haciendo uso de las bandas transportadoras, conformará la mejor retroalimentación a este estudio.

\section{Objetivo de la práctica}

- Analizar la estructura del producto requerido, simular una línea de producción y así mismo calcular el tiempo de ensamble para hacer un MRP.

\section{Recursos materiales}

- Banda transportadora

- Cronómetro

- Set de Lego

- $\quad$ MRP de práctica 8

- Balanceo de líneas práctica 4

- Estudio de tiempos práctica 2

\section{Desarrollo}

La cantidad de participantes requerida es: una persona por cada estación de trabajo de acuerdo con la información obtenida en la práctica 7, quienes fungirán como los operarios de cada estación, además de otro alumno con la función de abastecer a cada estación de trabajo con los materiales requeridos.

Acomodar las bandas transportadoras en línea y regular la velocidad de acuerdo con el tiempo ciclo y la distancia entre las estaciones de trabajo.

Simular la línea de ensamble de acuerdo con la distribución de operaciones del balanceo de líneas de la práctica 4. El encargado de abastecimiento proveerá a cada estación de trabajo con los materiales requeridos de acuerdo con el MRP. Realizar los ajustes necesarios para su correcto funcionamiento.

\section{Hacer una reflexión sobre los resultados obtenidos:}

- Cumplimiento de tiempos

- Niveles de inventario

- Flujo de la línea sin interrupciones

PRÁCTICA 10. Elaborar lista de inventario de materias primas con códigos de barras 


\section{Introducción}

Espinal \& Montoya (2009), definen al código de barras como una herramienta que sirve para capturar información relacionada con los números de identificación de artículos comerciales, unidades logísticas y localizaciones de manera automática, incluye el mejoramiento de la trazabilidad ${ }^{1}$. El código de barras es una serie de líneas paralelas y espacios de diferente grosor; el ancho de las líneas y de los espacios determina el dato codificado en el código, es decir, no contiene información, sólo identifica el producto.

\section{Objetivo de la práctica}

- El alumno conocerá la estructura de datos básicos a los productos para elaborar la lista de inventario y generará sus códigos de barras.

\section{Recursos materiales}

- Equipo de cómputo

- Excel

- Set de Lego

- Impresora

- Papel para imprimir

- Lector de códigos de barras

- Videos de apoyo:

1) https://www.youtube.com/watch?v=DA9wySbjNmA

2) https://www.youtube.com/watchv=UJqM8h9_Hjw\&feature=youtu.be

\section{Desarrollo}

Elaborar una tabla en Excel para la lista de inventario, tomando como referencia para la identificación de la información la tabla 6, anotar en la lista los materiales que conforman el set de Lego.

\section{Tabla 6}

Datos solicitados

\begin{tabular}{|l|l|}
\hline \multicolumn{1}{|c|}{ Encabezado } & \multicolumn{1}{c|}{ Detalle } \\
\hline GTIN & Código de barras del articulo comercial \\
\hline Nombre genérico & Nombre Funcional, no debe de dar ningún atributo del artículo \\
\hline
\end{tabular}

\footnotetext{
${ }^{1}$ Trazabilidad: Serie de procedimientos que permiten seguir el proceso de evolución de un producto en cada una de sus etapas.
} 


\begin{tabular}{|l|l|}
\hline \multicolumn{1}{|c|}{ Encabezado } & \multicolumn{1}{c|}{ Detalle } \\
\hline $\begin{array}{l}\text { Marca del } \\
\text { producto }\end{array}$ & Marca de mayor impacto comercial. \\
\hline Sub-Marca & Nombre para diferenciar una línea de artículos de otra \\
\hline Variantes & $\begin{array}{l}\text { Descripción del artículo que lo hace único y/o diferente (Modelo, } \\
\text { Estilo, Tipo, Sabor, Aroma, etc.) }\end{array}$ \\
\hline Color & $\begin{array}{l}\text { Campo a considerar cuando venga en la etiqueta la palabra "Color" } \\
\text { y se maneje GTIN por color. }\end{array}$ \\
\hline Talla & $\begin{array}{l}\text { Atributo relevante siempre y cuando se maneje GTIN por talla. } \\
\text { Solo se utiliza para ropa y calzado. }\end{array}$ \\
\hline Descripción & $\begin{array}{l}\text { Agrupación de los siguientes campos: } \\
\text { marca + submarca + nombre genérico + variantes }\end{array}$ \\
\hline Contenido Neto & $\begin{array}{l}\text { Declaración en el empaque. Si el artículo es un kit se realiza la } \\
\text { suma de los contenidos. Se declara en piezas }\end{array}$ \\
\hline
\end{tabular}

Fuente: GS1 México 2015

Para la columna de "Descripción”, utilizar la fórmula “concatenar" de Excel (ver tabla 7), en donde se seleccionarán las celdas correspondientes a: Nombre genérico, marca, submarca, variante, color y talla, en este caso no aplica talla para Lego.

\section{Tabla 7}

Fórmula para descripción

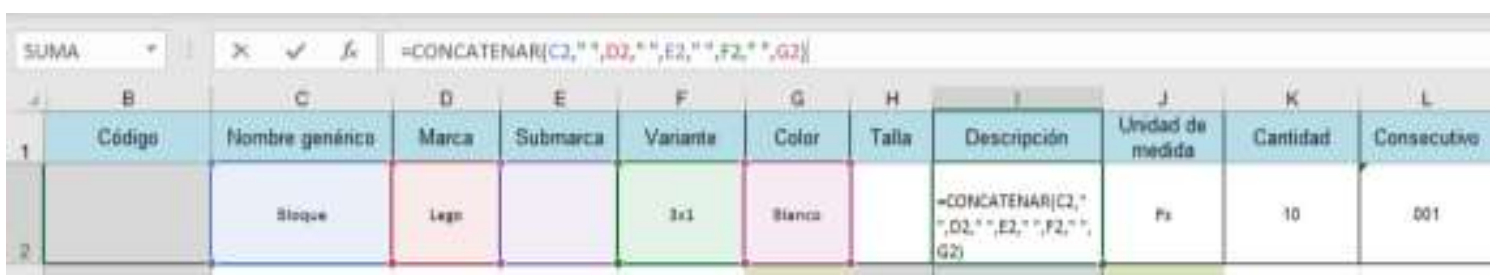

Posteriormente para generar el código se aplica la fórmula que se muestra en la tabla 8.

\section{Tabla 8}

Fórmula para código

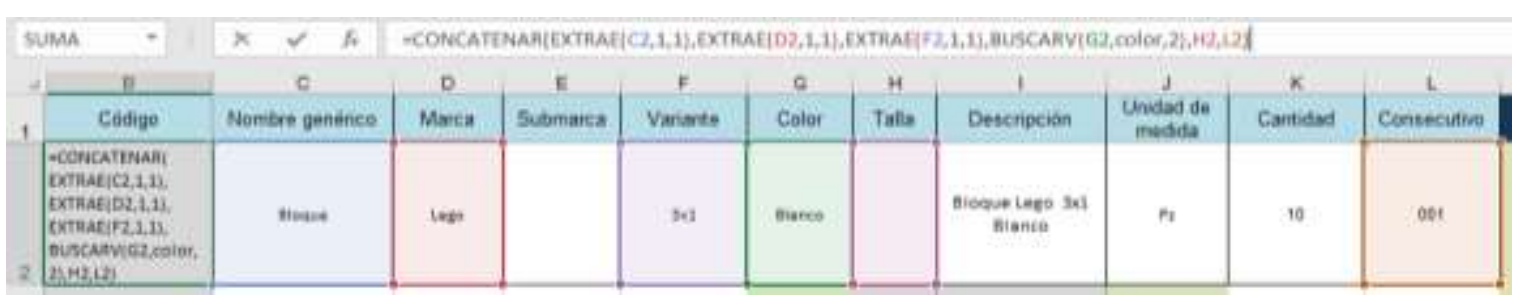

Descargar en Excel el tipo de fuente para los códigos de barras (video 1). 
Elaborar una tabla en Excel (ver Tabla 9), donde los códigos de la primer columna se establecerán de forma aleatoria, pueden ser códigos alfanuméricos.

\section{Tabla 9}

Lista de productos

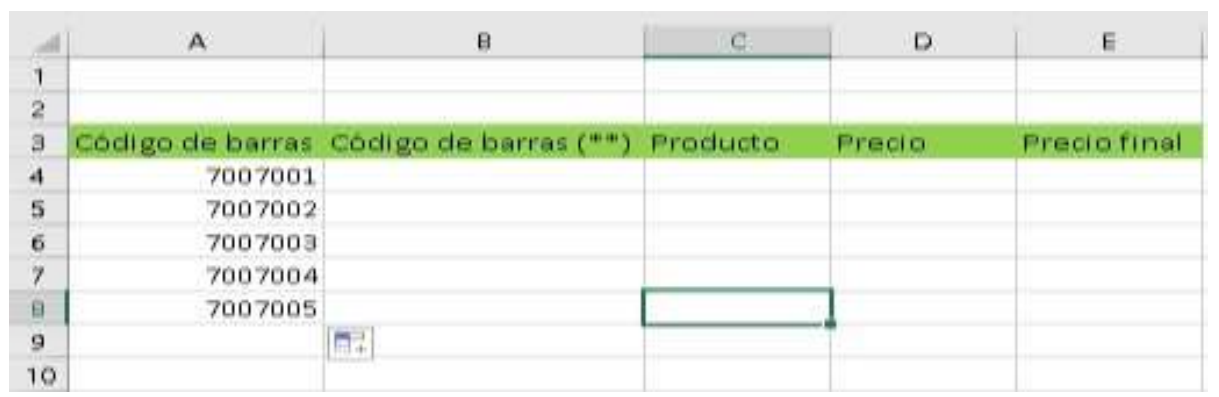

En la columna 2 introducir la siguiente fórmula y copiar la fórmula al resto de la columna, ver Tabla 10.

="*"\&[@[Codigo de barras]]\&"*" (La palabra códigos de barras en la fórmula seria A4),

\section{Tabla 10}

Fórmula para código de barras

\begin{tabular}{|c|c|c|c|c|c|}
\hline 4 & A & B & c & D & E \\
\hline \multicolumn{6}{|l|}{1} \\
\hline \multicolumn{6}{|l|}{2} \\
\hline 3 & Codigo de barras & Codigo de barras (**) & Producto & Precio & Precio final \\
\hline 4 & 7007001 & ="*"*[@[Codigo de ba & arras]]\&"**" & & \\
\hline 5 & 7007002 & *7007002* & & & \\
\hline 6 & 7007003 & *7007003* & & & \\
\hline 7 & 7007004 & *7007004* & & & \\
\hline 8 & 7007005 & $* 7007005 *$ & & & \\
\hline 9 & 7007006 & *7007006* & & & \\
\hline 10 & 7007007 & *7007007* & & & \\
\hline 11 & & & & & \\
\hline
\end{tabular}

Terminar de llenar la información de la tabla, ver ejemplo Tabla 11.

\section{Tabla 11}

Lista de productos completa

\begin{tabular}{|c|c|c|c|c|c|}
\hline 4 & A & B & c & D & E \\
\hline \multicolumn{6}{|l|}{1} \\
\hline \multicolumn{6}{|l|}{2} \\
\hline 3 & Codigo de barras & Codigo de barras (**) & Producto & Precio & Precio final \\
\hline 4 & $\begin{array}{r}7007001 \\
\end{array}$ & *7007001* & Playera & 150 & 150 pesos \\
\hline 5 & 7007002 & *7007002* & Gorras & 200 & 200 pesos \\
\hline 6 & 7007003 & *7007003* & Cubre bocas & 15 & 15 pesos \\
\hline 7 & 7007004 & *7007004* & Tenis & 1500 & 1500 pesos \\
\hline 8 & 7007005 & *7007005* & Pants & 300 & 300 pesos \\
\hline 9 & 7007006 & *7007006* & & & \\
\hline 10[ & 7007007 & *7007007* & & & \\
\hline 11 & & & & & \\
\hline
\end{tabular}


Generar una segunda hoja en el mismo archivo y elaborar el formato de etiqueta, como lo muestra la figura 1, donde se copiará la información contenida en la tabla anterior.

\section{Figura 1}

Diseño de etiqueta

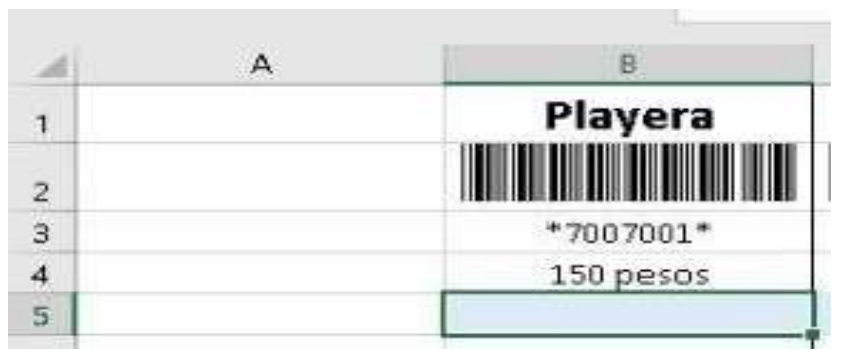

Se pueden generar las etiquetas para los demás productos de la lista, copiando las fórmulas hacia la derecha y hacia abajo, procurando aprovechar el espacio de la hoja, ver figura 2, e imprimir la hoja.

\section{Figura 2}

Etiquetas de la lista

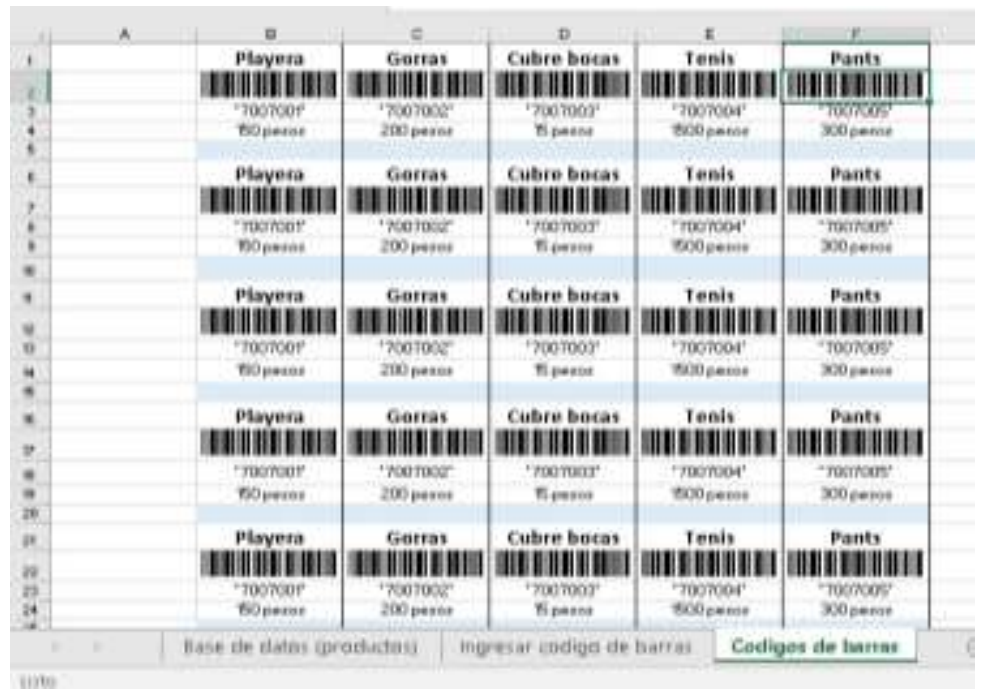

Escanear código impreso y comprobar lo reconozca el Excel.

\section{CONCLUSIÓN O CONSIDERACIONES FINALES}

El manual de prácticas de laboratorio de tecnologías aplicadas a la logística no sólo es de beneficio para los estudiantes de la Ingeniería en Logística y Transporte, sino que es un espacio para que todas aquellas personas relacionadas con la logística puedan realizar simulaciones de trabajo y desarrollar estrategias nuevas de mejora al hacer uso de los diversos equipos y metodologías para trabajo en conjunto. 


\section{LISTA DE REFERENCIAS}

Blandón, M. G. (2021). Estudio de caso: Desarrollo de una metodología (DMAIC o Seis Sigma) para evaluación de mermas de materia prima en la planta de alimentos balanceados de la Escuela Agrícola Panamericana, Zamorano.

Chase, R. B., Jacobs, R. \& Aquilano, N. J. (2009). Administración de operaciones Producción y cadena de suministros. México: Mcgraw-hill.

Criollo, R. G. (2005). Estudio del trabajo Ing. de métodos y medición del trabajo. México: Mc Graw-Hill.

ESPINAL, A. C., \& Montoya, R. A. G. (2009). Tegnologías de la información en la cadena de suministro. Dyna, 76(157), 37-48.

Espinosa, E., A., González, K. D. \& Hernández, L. (2016) Las prácticas de laboratorio: una estrategia didáctica en la construcción de conocimiento científico escolar, 12(1), 266-281. ISSN 19003803. https://doi.org/10.18041/entramado.2016v12n1.23125.

EXACTAS, Á. D. C. (2010). MANUAL DE PRÁCTICAS Y ANTOLOGÍA (Doctoral dissertation, BENEMÉRITA UNIVERSIDAD AUTÓNOMA DE PUEBLA).

Fernández Collado, C., Baptista Lucio, P., \& Hernández Sampieri, R. (2014). Metodología de la Investigación. Editorial McGraw Hill.

Insausti, L. H. (2020). Prácticas de laboratorio en el proceso de Enseñanza-Aprendizaje de la asignatura de Biología en el segundo año de bachillerato general unificado del Instituto Nacional

Kanawaty, G. (1996). Introducción al estudio del trabajo OIT. Ginebra:. PÁGINA 157158

Marín, M. (2008). El trabajo experimental en la enseñanza de la química en contexto de resolución de problemas en el laboratorio. Un caso particular la combustión. En: Maestría en Educación énfasis Enseñanza de las Ciencias Naturales. Universidad del Valle.

Niebel, B., \& Freivalds, A. (2009). Métodos, estándares y diseño del trabajo. Onceava ed, 1 .

Pinheiro de Lima, O., Breval Santiago, S., Rodríguez Taboada, C. M., \& Follmann, N. (2017). Una nueva definición de la logística interna y forma de evaluar la misma. Ingeniare. Revista chilena de ingeniería, 25(2), 264-276. 
Rodríguez-Gómez, D., Armengol, C., \& Meneses, J. (2017). La adquisición de las competencias profesionales a través de las prácticas curriculares de la formación inicial de maestros. Revista de Educación, 376, 229243. https://doi.org/10.4438/1988-592X-RE-2017-376-350

Rodríguez, C. R. C., \& León, C. A. M. (s.f.). SISTEMA DE PLANIFICACIÓN DE LOS REQUERIMIENTOS MATERIALES (MRP).

Salazar, B. (2019). Cálculo del número de observaciones. Abril 8,2021, de Ingenieria Indistrial Online Sitio web: https://www.ingenieriaindustrialonline.com/estudiode-tiempos/calculo-del-numero-de-observaciones/

Sorensen, P. (2014). Collaboration, dialogue and expansive learning: Theuse of paired and multiple placements in the school practicum.Teaching and Teacher Education, 44, 128137.doi:http://dx.doi.org/10.1016/j.tate.2014.08.

Suárez, J. D. A., \& Mendoza, M. A. M. (2006). Guía de elaboración de un manual de prácticas de laboratorio, taller o campo: asignaturas teórico-prácticas. México: Universidad Autónoma Chapingo.

UPMH. (2021). Acerca de nosotros. agosto 4, 2021, de SEPH Sitio web: http://www.upmetropolitana.edu.mx/acerca-de 